\title{
Apoio social em trabalhadores da saúde: tendências das produções nacionais
}

\author{
Social support in health workers: trends in national productions \\ Apoyo social en los trabajadores sanitarios: tendências en las producciones nacionales
}

Marculina da Silva

ORCID: https://orcid.org/0000-0001-6106-0582 Universidade Federal de Santa Maria, Brasil E-mail: marculinasilva30@gmail.com

Rafaela Andolhe

ORCID: https://orcid.org/0000-0003-3000-8188 Universidade Federal de Santa Maria, Brasil E-mail: rafaela.andolhe@ufsm.br

Mauren Pimentel Lima

ORCID: https://orcid.org/0000-0002-0215-1190 Universidade Federal de Santa Maria, Brasil E-mail: maurenplima@gmail.com

Laura Prestes Moreira

ORCID: https://orcid.org/0000-0002-2577-0832 Universidade Federal de Santa Maria, Brasil E-mail: prestesmoreira96@gmail.com

Tainá Caroline Gonçalves de Souza ORCID: https://orcid.org/0000-0001-9469-3116 Universidade Federal de Santa Maria, Brasil E-mail: tainacgs@ hotmail.com

Tânia Solange Bosi de Souza Magnago ORCID: https://orcid.org/0000-0002-5308-1604 Universidade Federal de Santa Maria, Brasil E-mail: magnago.tania@gmail.com

Adriel padilha

ORCID: https://orcid.org/0000-0002-5229-5811 Universidade Federal de Santa Maria, Brasil E-mail: adrielpadilha00@gmail.com

\begin{abstract}
Resumo
Objetivo: identificar as tendências das produções científicas de teses e dissertações nacionais em relação ao apoio social em trabalhadores da saúde. Método: trata-se de uma revisão narrativa da literatura, realizada no Catálogo de Teses e Dissertações da Coordenação e Aperfeiçoamento de Pessoal de Nível Superior. A busca foi realizada em julho de 2021, através de estratégia, "apoio social" AND trabalhadores de saúde. Sem recorte temporal. A busca resultou em 115 produções e destas foram incluídos 08 estudos. Resultados: das oito produções incluídos no estudo, 100\% $(n=08)$ eram dissertações. A partir disso, distinguiu-se duas categorias para análise e discussão: caracterização das produções e tendência em relação ao apoio social. Identificou-se o impacto do apoio social na saúde do trabalhador em seu ambiente laboral e sua correlação com sofrimento, adoecimento, estresse, presenteísmo e intenção de abandonar a profissão. Considerações finais: evidenciou tendências da relação do apoio social com à saúde do trabalhador, pois este atua como protetor da saúde sob efeitos lesivos quando presente no ambiente do trabalho. Observou-se que quando se encontra baixo, pode potencializar o desenvolvimento dos agravos relacionados a labor e diminuição da qualidade de vida dos profissionais. Os resultados deste estudo proporcionaram identificar que ainda há lacunas científicas oriundas das produções nacionais acerca do apoio social entre trabalhadores de atenção primária, sendo uma área importante de investigação. Verifica-se a relevância de pesquisas abordarem a temática nesse contexto, contribuindo para a saúde do trabalhador, com vistas ao desenvolvimento de estratégias para promover ações que fortalecem o apoio social.
\end{abstract}

Palavras-chave: Saúde do trabalhador; Apoio social; Trabalhadores de saúde; Literatura de revisão.

\begin{abstract}
Objective: to identify trends in national scientific productions of theses and dissertations regarding social support in health care workers. Method: this is a narrative review of the literature, carried out in the Catalog of Theses and Dissertations of the Coordination for the Improvement of Higher Education Personnel. The search was performed in July 2021, through strategy, "social support" and health workers. No time cut. The search resulted in 115 productions, of which 08 studies were included. Results: of the eight productions included in the study, $100 \%$ ( $\mathrm{n}=08$ ) were
\end{abstract}


dissertations. From that, we distinguished two categories for analysis and discussion: product characterization and trends regarding social support. The impact of social support on the health of workers in their work environment and its correlation with suffering, illness, stress, presenteeism and intention to leave the profession were identified. Final considerations: It evidenced tendencies of the relation of social support with the worker's health, since this acts as a health protector under harmful effects when present in the work environment. It was observed that when it is low, it can potentiate the development of work-related diseases and decrease the quality of life of professionals. The results of this study allowed us to identify that there are still scientific gaps in the national productions about social support among primary care workers, being an important area of investigation. It is important to address the theme in this context, contributing to the health of the worker, with a view to developing strategies to promote actions that strengthen social support.

Keywords: Worker's health; Social support; Health care workers; Literature review.

\begin{abstract}
Resumen
Objetivo: identificar las tendencias de las producciones científicas de las tesis y disertaciones nacionales en relación con el apoyo social a los trabajadores sanitarios. Método: se trata de una revisión narrativa de la literatura, realizada en el Catálogo de Tesis y Disertaciones de la Coordinación de Perfeccionamiento de Personal de Nivel Superior. La búsqueda tuvo lugar en julio de 2021, mediante la estrategia, "apoio social" AND trabajadores sanitarios. No hay corte de tiempo. La búsqueda dio como resultado 115 producciones; y, de ellas, se incluyeron 08 estudios. Resultados: de las ocho producciones incluidas en el estudio, el 100\% $(n=08)$ eran disertaciones. A partir de ello, se distinguieron dos categorías para el análisis y la discusión: la caracterización de las producciones y las tendencias relativas al apoyo social. Se identificó el impacto del apoyo social a la salud de los trabajadores en su entorno laboral y su correlación con el sufrimiento, la enfermedad, el estrés, el presentismo y la intención de abandonar la profesión. Consideraciones finales: se evidenciaron las tendencias de la relación del apoyo social con la salud del trabajador, ya que este actúa como protector de la salud frente a los efectos perniciosos cuando está presente en el entorno laboral. Se observó que cuando se encuentra bajo, puede potencializar el desarrollo de los males relacionados con el trabajo y la disminución de la calidad de vida de los profesionales. Los resultados de este estudio identificaron que todavía existen lagunas científicas en las producciones nacionales sobre el apoyo social entre los trabajadores de la atención primaria, lo que constituye un área importante de investigación. Se nota que es relevante que la investigación aborde el tema en este contexto, contribuyendo a la salud del trabajador, con miras a desarrollar estrategias para promover acciones que fortalezcan el apoyo social.
\end{abstract}

Palabras clave: Salud laboral; Apoyo social; Personal de salud; Literatura de Revisión.

\title{
1. Introdução
}

O trabalho apresenta-se como um importante aspecto que atribui a valorização do status pessoal, geração de renda e propicia a inclusão social dos que trabalham, auxiliando a formação de redes de apoio sociais fundamentais para a saúde, impulsionando um efeito protetor que promove a saúde. Em contrapartida, os riscos gerados em processos produtivos em um local de trabalho com condições estressantes e desgastantes, que não garantem segurança à saúde do trabalhador, podem ocasionar perturbação e sofrimento aos trabalhadores (Brasil, 2001; Brasil, 2018).

Nesta perspectiva, a saúde do trabalhador, como sendo um campo da saúde pública, possui como objeto de estudo e ações, as relações produção-consumo e o processo saúde-doença dos indivíduos, em especial dos trabalhadores. Desta forma, o trabalho pode ser considerado como base organizadora da vida social, área de dominação, resistência dos trabalhadores, determinante das condições de vida e saúde das pessoas. Sendo assim, as ações das organizações de trabalho em geral devem procurar a mudança dos processos produtivos, de modo a torná-los propulsores de saúde, mitigando o risco de adoecimento e morte, e também assegurar a atenção integral à saúde dos trabalhadores, considerando sua inserção nos processos produtivos (Brasil, 2018).

Logo, o contexto de trabalho, pode ser adverso aos trabalhadores decorrente das situações distintas que os posicionam em uma grande pressão, dentre elas destacam-se: a sobrecarga e exigências do trabalho, condições precárias do trabalho, ritmo laboral acelerado, exposição aos fatores de risco a saúde, falta de suporte social informacional, aspectos que impactam no desempenho das tarefas, associado a isto, também há dificuldade na relação interpessoais e o baixo reconhecimento atrelado a depreciação profissional. Assim, tais aspectos têm uma relação intrínseca com fatores relacionados à organização, processo de 
trabalho e principalmente ao relacionamento interpessoal, que possam impactar negativamente a saúde dos trabalhadores (Junior et al., 2014).

Nesse sentido, o apoio social foi uma das dimensões incorporadas ao modelo de demanda-controle de Karasek e Theorell, sua inserção foi proposta por Jeffrey Johnson em 1988, pelo fato deste considerá-lo uma necessidade humana que pode configurar como um aspecto que interfere diminuindo o impacto gerado pela demanda excessiva no trabalho. Ou seja, acredita-se que sua adição pode ampliar a formulação de controle de demanda por meio de uma ênfase na conexão individual entre pessoas e seu trabalho, numa relação coletiva.

Diante do exposto, o apoio social compreende o nível de interação social presente no trabalho, com supervisores e colegas de trabalho no contexto das organizações (Esteves et al., 2019). Além disso, este se associa aos aspectos das relações sociais que conferem um efeito sobre a saúde física e mental das pessoas (Fonseca \& Moura, 2008). Nesse sentido, o apoio social caracteriza-se como um construto capaz de atenuar a percepção das ameaças, funcionando como um recurso ambiental importante no processo de enfrentamento das situações adversas (Matos et al., 2017), sendo este considerado por Karasek e Theorell (1990) como um aspecto relevante para as condições precárias nas situações desgastantes que estão acima do controle dos riscos pelos trabalhadores.

Conceitua-se o apoio social como auxílio material, informações oferecidas por grupos ou pessoas que se conhecem, resultando em efeitos emocionais e/ou comportamentais positivos. Sendo este um processo recíproco que gera efeitos positivos para o receptor, bem como para quem oferece o apoio, permitindo que ambos tenham o controle da situação (Chor et al., 2001; Andrade \& Pérez-Nebra, 2017; Andrade, \& Vaitsman, 2002).

Nesta perspectiva, a saúde dos trabalhadores pode estar diretamente associada ao suporte institucional recebido por eles no âmbito laboral, em que quanto maior o suporte, menor o estresse ocupacional, derivando em consequência, no aperfeiçoamento da saúde mental, com o risco reduzido ao desenvolvimento de problemas psicológicos (Bertoncello et al., 2017). Em contrapartida, ressalta-se que o suporte social baixo possui relação intrínseca com perturbações do trabalho, pois este pode possibilitar o aumento do risco de pessoas serem acometidas por estresse psicossocial, comprometendo deste modo, sua saúde. Reforça-se a relevância de manutenção de apoio social por parte de gestores e organizações de saúde (Fernandes et al., 2018; Fernandes, 2016).

O estudo apresenta sua pertinência, devido à necessidade de ter a ciência da produção sobre o assunto de apoio social. E assim, proporcionar a noção das possíveis lacunas existentes quanto ao tema em questão e ampliar a sua investigação.

Desta forma, partiu-se da seguinte questão da pesquisa: "Quais as tendências das produções nacionais de teses e dissertações brasileiras acerca do apoio social em trabalhadores da saúde?"

Assim, o presente estudo teve como objetivo: Identificar as tendências das produções científicas de teses e dissertações nacionais em relação ao apoio social em trabalhadores da saúde.

\section{Metodologia}

O estudo trata-se de uma revisão narrativa da literatura (RNL), descritivo e de caráter qualitativo. A RNL proporciona caracterização das produções sobre um determinado assunto, bem como investigar assuntos amplos que destacam tendências, e produção integral em relação ao tema pretendido. Além disso, assegura ao pesquisador o contato e conhecimento inicial com o objeto de estudo, por meio de uma extensa busca do fato a ser investigado (Brum et al., 2016). Realizou-se uma busca de dados em julho de 2021, no Catálogo de Teses e Dissertações da Coordenação e Aperfeiçoamento de Pessoal de Nível Superior (CAPES). 
A partir disso, foram adotados como critério de inclusão: teses e dissertações disponíveis na íntegra acerca do suporte social em trabalhadores da saúde. Foram excluídos: estudos que não respondessem a questão e objetivo da revisão. Não foi especificado o recorte temporal, de modo a alcançar maior número de produções.

Definiram-se como estratégia de busca os seguintes termos: "apoio social" AND trabalhadores de saúde". O resultado da busca foi apresentado no fluxograma (Figura 1).

Para posterior análise dos dados de estudos, utilizou-se a ferramenta Excel, para organização destes em quadro sinóptico contendo autor/ano, modalidade (dissertação ou tese), instituição de ensino, delineamento do estudo, população, cenário, principais resultados e conclusões.

Os dados do presente estudo foram interpretados por meio de uma abordagem qualitativa e com uma análise crítica pessoal e apresentados por meio de duas categorias, sendo que este tratou-se de uma revisão narrativa.

No que concerne às questões éticas no tratamento dos dados das produções, resultados obtidos pelos autores dos estudos selecionados para a análise, foram reproduzidos com autenticidade, sem sofrer alterações. Em seguida, serão apresentadas as categorias.

\section{Resultados e Discussão}

Como resultado da busca, foram obtidos 115 estudos. Após a leitura de títulos e resumos, baseados no critério de inclusão e exclusão, foram excluídos (107) estudos. Sendo os que não atenderam a temática e/ou pergunta da pesquisa (92) estudos e por não estarem disponíveis na íntegra (15) estudos. Desta forma, constitui-se o corpus final da revisão, um total de (08) estudos. Conforme demonstrado no fluxograma (Figura 1).

Figura 1: Fluxograma das produções selecionado para estudo. Santa Maria/RS. 2021.

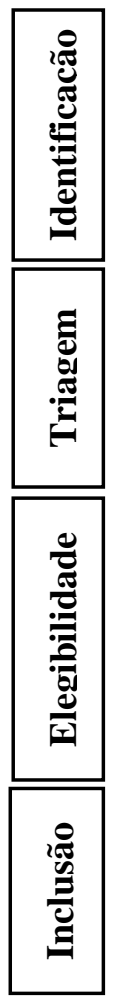

Estudos identificados na busca no catálogo de Teses e Dissertações da CAPES

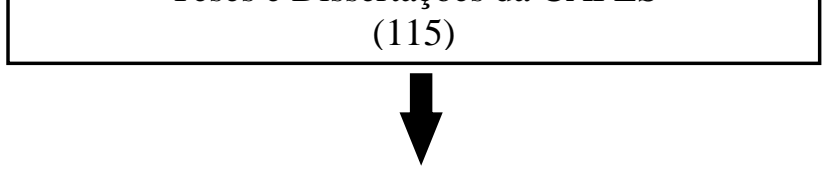

Leitura de títulos e resumos

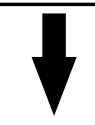

Excluídos (107)

Por não atenderam temática e/ou pergunta da pesquisa $=92$

Por não estarem disponível na íntegra $=15$

Fonte: Dados do estudo (2021). Fluxograma adaptado com base em Carneiro et al., (2020). 
Por meio da leitura, análise e caracterização das produções selecionadas, tornou-se possível evidenciar as tendências nacionais acerca da temática de apoio social dos trabalhadores de saúde, assim como, apresentar as categorias para análise e discussão.

Primeiramente será apresentado a caracterização das produções e, em seguida a tendência do apoio social segundo Modelo demanda-controle com ênfase na dimensão apoio social de Karasek e Theorell (1990).

\section{Caracterização dos estudos}

Concernente aos 08 estudos incluídos na revisão, $100 \%$ (n=08) eram dissertações. Quanto ao ano de publicação das produções, houve uma variação entre 2014 a 2020. Em 2015; 2016; 2017 e 2020 obteve-se 12,50\% (n=01). Sendo os anos com maiores produções foram 2014 e 2018 com 25\% (n=02), acredita-se que isso se deve a preocupação da condição que gira em torno da saúde física e mental gerada nas organizações de trabalho associado a forma de produção de capital que acaba impactando na capacidade da tomada de decisão dos trabalhadores (Baglioli, 2017). Enfatizando assim a busca de meios para auxiliar no enfrentamento das exigências e pressão psicológicas que as demandas excessivas de trabalho impõem nos profissionais no ambiente laboral, gerando desse modo o estresse.

Referente ao delineamento das produções, $100 \%(\mathrm{n}=08)$ eram do tipo quantitativos, com base em Bezerra e Neves (2010) esse aumento das produções com delineamento quantitativo se deve a crescente interação da epidemiologia com a área da saúde do trabalhador. A pesquisa quantitativa possibilita uma análise dos dados de modo concreto por meio da estatística para a interpretação dos resultados colhidos para executar a correlação da realidade empírica com a teoria que embasa o estudo (Silva, 2010; Munhoz et al., 2018; Polit \& Beck, 2019).

No que se refere às instituições de proveniência das produções, houve predomínio das instituições da região sudeste do Brasil com 50\% ( $\mathrm{n}=04)$. Destaca-se que tal predomínio decorre dos seguintes aspectos: maior quantidade de grupos vinculados à pesquisa, bem como o número dos pesquisadores, como também a questão dos programas de pós-graduação e periódicos voltados à publicação de artigos relacionados à saúde, nessa região (Bezerra \& Neves, 2010; Sidone, Haddad \& Mena-Chalco, 2016).

Em relação a população, observa-se que predominou os trabalhadores da enfermagem com $75 \%(n=6)$. Fato que pode ser justificado pelo contato direto com o paciente que esta categoria tem durante assistência à saúde e encontram-se exposto a diversos riscos ligado a atividade laboral desempenhado muitas vezes, em condições adversas e de muito desgaste (Filho \& Almeida, 2016), despertando a necessidade de estudar essa população. Outra categoria analisada nas produções é o Agente comunitária da saúde (ACS), com 25\% (n=02).

No tocante dos cenários das produções, verificou-se maior concentração de estudos na área hospitalar com 62,50\% $(\mathrm{n}=05)$. Tal fato pode estar relacionado ao uso da alta tecnologia para desenvolvimento de assistência em saúde, em que os profissionais lidam com as necessidades complexas dos usuários, ou seja, local no qual, o cuidado é crítico (Silva \& Silva, 2019). No que se refere ao cenário do estudo, constatou-se um percentual baixo para Atenção Primária à Saúde (APS), totalizando $37,50 \%$ ( $\mathrm{n}=03$ ), indicando uma lacuna quanto ao tema.

No Quadro 1 é apresentada as Caracterização das produções. 
Quadro 1: Características das produções selecionadas para revisão por meio de Catálogo de Teses e Dissertações da Coordenação e Aperfeiçoamento de Pessoal de Nível Superior (CAPES). (N=08) Santa Maria, RS. 2021.

\begin{tabular}{|c|c|c|c|c|c|c|}
\hline ID & Autor/Ano & Modalidade & Instituição de ensino & $\begin{array}{l}\text { Delineamento do } \\
\text { estudo }\end{array}$ & População & Cenário \\
\hline P1 & Fernandes (2016) & Dissertação & $\begin{array}{l}\text { Universidade Federal de Juiz de } \\
\text { Fora }\end{array}$ & Quantitativo, transversal & ACS & APS \\
\hline $\mathbf{P 2}$ & Velasco (2014) & Dissertação & $\begin{array}{l}\text { Universidade Federal do Estado } \\
\text { do Rio de Janeiro }\end{array}$ & Seccional, quantitativa & $\begin{array}{l}\text { Trabalhadores de } \\
\text { enfermagem }\end{array}$ & APS \\
\hline P3 & Candaten (2015) & Dissertação & $\begin{array}{l}\text { Universidade Federal do Rio } \\
\text { Grande do Sul }\end{array}$ & Quantitativo transversal & Enfermeiros & Hospital \\
\hline $\mathbf{P 4}$ & Coimbra (2017) & Dissertação & Universidade de Sagrado Coração & Quantitativo & $\begin{array}{l}\text { Profissionais } \\
\text { enfermagem }\end{array}$ & Hospital \\
\hline P5 & Rocha (2018) & Dissertação & $\begin{array}{l}\text { Universidade Federal de Mato } \\
\text { Grosso }\end{array}$ & $\begin{array}{l}\text { Epidemiológico, } \\
\text { transversal }\end{array}$ & Enfermeiros & Hospital \\
\hline P6 & Belini (2018) & Dissertação & $\begin{array}{l}\text { Universidade Federal De Juiz De } \\
\text { Fora }\end{array}$ & $\begin{array}{l}\text { Epidemiológico } \\
\text { descritivo, seccional }\end{array}$ & ACS & APS \\
\hline P7 & Silva (2014) & Dissertação & $\begin{array}{l}\text { Universidade Católica Dom } \\
\text { Bosco }\end{array}$ & $\begin{array}{l}\text { Epidemiológico, } \\
\text { transversal }\end{array}$ & $\begin{array}{l}\text { Profissionais } \\
\text { enfermagem }\end{array}$ & Hospital \\
\hline P8 & Gadelha (2020) & Dissertação & Universidade Federal do Acre & $\begin{array}{l}\text { Epidemiológico, } \\
\text { transversal, quantitativo }\end{array}$ & Enfermeiros & Hospital \\
\hline
\end{tabular}

Fonte: Dados do estudo (2021). Elaboração própria.

Tendências das produções quanto ao apoio social segundo modelo demanda-controle com ênfase no apoio social.

Neste tópico do estudo é abordado a questão do suporte social, de acordo com modelo de demanda-controle e apoio social de Karasek e Theorell (1990), com base nos resultados e conclusões das produções selecionadas. A partir da análise dos estudos, foi possível apontar as tendências nacionais em relação à temática em questão. No Quadro 2 são apresentados os principais resultados e conclusões encontrados nas produções selecionados para revisão. 
Quadro 2: Quadro sinóptico. Principais resultados e conclusões encontrados nos estudos selecionados para revisão, por meio de Catálogo de Teses e Dissertações da Coordenação e Aperfeiçoamento de Pessoal de Nível Superior (CAPES). Santa Maria, RS. 2021.

\begin{tabular}{|c|c|c|}
\hline ID & PRINCIPAIS RESULTADOS & CONCLUSÃO \\
\hline P1 & $\begin{array}{l}\text { Maior proporção dos trabalhadores na categoria de trabalho de } \\
\text { alta exigência, que é grupo de maior exposição para } \\
\text { desenvolver o estresse psicossocial no trabalho e aspecto } \\
\text { importante dos riscos ao estresse. Ou seja, a maioria dos } \\
\text { trabalhadores apresentaram alta demanda, baixo controle e alto } \\
\text { apoio social. }\end{array}$ & $\begin{array}{l}\text { Conclui-se que maior proporção dos indivíduos nos trabalhos passivo e alta } \\
\text { exigência, o que gera alto risco de acometimento em saúde e desenvolvimento do } \\
\text { estresse psicossocial no trabalho. Ressalta-se a importância de apoio social por } \\
\text { parte dos gestores e colaboradores, pois presença de alto apoio social, pode agir de } \\
\text { modo a minimizar ou proteger os trabalhadores dos efeitos negativos advindos da } \\
\text { exposição a agentes estressores. }\end{array}$ \\
\hline P2 & $\begin{array}{l}30,8 \% \text { dos trabalhadores se enquadram na baixa demanda e } \\
\text { baixo controle, classificados no quadrante trabalho passivo, } \\
\text { sinalizando uma situação desestimulante, tediosa. 55\% } \\
\text { apresentam o risco de distúrbios psicológicos e de doença } \\
\text { física, pois trabalho é de alta demanda e baixo controle. A } \\
\text { maioria dos trabalhadores possui apoio social baixo, } \\
\text { propiciando o estresse ocupacional. }\end{array}$ & $\begin{array}{l}\text { Desvelou-se que a analogia existente entre o risco de distúrbios psicológicos e } \\
\text { doenças físicas e o apoio social baixo, predispõe ao estresse laboral. São } \\
\text { preocupantes, pois demonstraram a existência de trabalhadores com risco de } \\
\text { adoecimento físico e psíquico atuando na estratégia saúde da família, tais } \\
\text { adoecimento podem expandir se as condições laborais não aprimorarem. } \\
\text { Importância de trabalhador reconhecer risco laborais, para prevenção do agravo e } \\
\text { promoção da saúde. }\end{array}$ \\
\hline P3 & $\begin{array}{l}\text { Percentual de enfermeiros sob estresse atingiu } 45,3 \% \text { da } \\
\text { amostra, verificou correlação entre demanda e apoio social, } \\
\text { quanto menor apoio social, maior demanda, vice-versa, } \\
\text { correlação inversa entre apoio social e intenção de abandonar } \\
\text { a profissão de enfermagem, evidenciou que quanto maior o } \\
\text { apoio social menor a despersonalização. E elevação de } \\
\text { cortisol. }\end{array}$ & $\begin{array}{l}\text { Salienta-se fortalecimento de vínculos interpessoais e desenvolvimento de } \\
\text { habilidades e competências que favorecem o enfrentamento do estresse laboral em } \\
\text { enfermeiros, promovendo qualidade de vida do trabalhador. }\end{array}$ \\
\hline $\mathbf{P 4}$ & $\begin{array}{l}\text { Grupos estudados apresentaram alta demanda, grupo } 1 \text {, com } \\
\text { média de } 14,1 \text { e grupo } 2 \text {, de } 14,5 \text {. Apoio social com bom nível, } \\
\text { grupo } 1 \text {, média } 18,4 \text { e grupo } 2,19,7 \text {. E controle com nível } \\
\text { ideal, grupo } 1 \text { média de } 17,7 \text { e grupo } 2 \text {, de } 18,0 \text {. Bom nível de } \\
\text { interação social, com média de } 32,9 \text { e } 40,4 \text { do ICT do grupo } 1 \\
\text { e } 2 \text { respectivamente. }\end{array}$ & $\begin{array}{l}\text { Resultado permitiram observar que apesar de profissionais terem demanda alta, } \\
\text { com média que indicaram pressões psíquicas, variáveis controle e apoio social } \\
\text { mantiveram no ideal, fato que indicou que os mesmos usam suas habilidades } \\
\text { intelectuais para tomada de decisão e obtendo bom nível de interação social. }\end{array}$ \\
\hline P5 & $\begin{array}{l}\text { Houve prevalência de trabalhadores que possuem alta } \\
\text { demanda no trabalho } 53(62,2 \%) \text {, e alto controle } 51(60 \%) \text {, o } \\
\text { que caracterizou no geral, um trabalho ativo. O apoio social } \\
\text { mostrou-se alto, com } 48(52,2 \%) \text { entre os trabalhadores. }\end{array}$ & $\begin{array}{l}\text { Estudo traz contribuições relevantes, pois ao caracterizar as condições de trabalho } \\
\text { por meio dos prognosticadores que definem a relação demanda, controle e apoio } \\
\text { social, permite que os gestores e os próprios trabalhadores busquem medidas para } \\
\text { amenizá-los e, assim melhorar a qualidade de vida no trabalho e assistência aos } \\
\text { pacientes. }\end{array}$ \\
\hline P6 & $\begin{array}{l}\text { Sobre o estresse psicossocial, } 32,5 \% \text { se encontravam na } \\
\text { categoria de alta exigência no trabalho, } 58,8 \% \text { da população } \\
\text { relataram ser presenteístas, o baixo apoio social teve } \\
\text { associação com o presenteísmo. }\end{array}$ & $\begin{array}{l}\text { Pode-se concluir que as hipóteses testadas foram comprovadas. A prevalência de } \\
\text { presenteísmo entre os agentes comunitários é maior que } 20 \% \text {, o presenteísmo } \\
\text { interfere negativamente na qualidade do trabalho e tem correlação com fatores } \\
\text { ocupacionais. }\end{array}$ \\
\hline P7 & $\begin{array}{l}\text { Em relação ao estresse no trabalho, } 60,8 \% \text { dos participantes } \\
\text { vivenciam uma alta demanda no trabalho, } 71,8 \% \text { um alto } \\
\text { controle sobre a atividade desempenhada, } 85,5 \% \text { baixo apoio } \\
\text { social e } 44,5 \% \text { vivenciam um trabalho ativo. }\end{array}$ & $\begin{array}{l}\text { Embora a maioria dos participantes da amostra vivencie uma situação de risco } \\
\text { intermediário para o estresse, a sua qualidade de vida relacionada à saúde } \\
\text { mostrou-se comprometida, apontando para o risco de adoecimento, bem como } \\
\text { para o desgaste físico e mental. }\end{array}$ \\
\hline P8 & $\begin{array}{l}\text { Sintomas depressivos apresentaram prevalência de } 25,8 \% \text { e } \\
\text { ansiedade de } 35,2 \% \text {. Foi identificada associação positiva de } \\
\text { sintomas depressivos com a qualidade do sono, apoio social, } \\
\text { trabalho ativo. }\end{array}$ & $\begin{array}{l}\text { Salienta-se a importância da implementação de estratégias de nível individual e } \\
\text { coletivo que visem minimizar esses efeitos na saúde dos trabalhadores }\end{array}$ \\
\hline
\end{tabular}

Fonte: Dados do estudo (2021). Elaboração própria.

Com base no Quadro 2, a síntese dos dados de estudos incluídos nesta revisão, possibilitou a observação das tendências acerca da relação e o impacto que o construto apoio social possui no âmbito laboral dos trabalhadores de saúde, pois suporte social baixo, frente a alta exigência do labor, pode acarretar no sofrimento e adoecimento dos profissionais.

No que concerne as tendências dos estudos selecionados, nas P1, P3, P4 e P5, os autores evidenciaram que entre os trabalhadores que apresentaram o apoio social alto, observou-se que este atua como um fator de proteção que atenua os efeitos deletérios do estresse ocupacional. Além disso, as tendências evidenciaram a correlação inversa do apoio social com a intenção de abandonar a profissão, também se ressalta que o apoio social auxilia os trabalhadores no uso de suas habilidades para a tomada de decisões em funções que desempenham, mesmo frente das demandas excessivas de trabalho (Fernandes, 2016; Candaten, 2015; Coimbra, 2017; Rocha, 2018).

Por outro lado, entre os trabalhadores que apresentaram baixo apoio social, como evidenciado nas P2, P6, P7 e P8, houve a predisposição ao estresse, impactando no distúrbios psicológicos e doenças físicas, bem como presenteísmo, risco para 
o adoecimento, desgaste físico e mental, depressão e qualidade de sono (Velasco, 2014; Belini, 2018; Silva, 2014; Gadelha, 2020).

O apoio social se configura como um aspecto protetor das possíveis condições dos efeitos deletérios e desgastantes que o trabalhador não consegue controlar. Pode ainda funcionar como um recurso ambiental essencial de tais situações (Karasek \& Theorell 1990; Matos et al., 2017).

Sendo um aspecto psicossocial essencial em todas as organizações de trabalho, o apoio social apresenta relevância no que concerne ao trabalho em saúde, devido a repercussão que possui na relação interpessoal presente no âmbito laboral dos trabalhadores. Além disso, frente aos problemas de saúde apresentados pela comunidade, tal construto potencializa a competência dos profissionais de saúde na sua resolução e solidariedade nas situações adversas. Promove também a cooperação entre a equipe, com o propósito de melhorar assistência à saúde, bem como supressão dos fatores agravantes à saúde do trabalhador (Matos et al., 2017).

O nível alto de apoio social, foi destacado nos estudos como fator que impacta positivamente na saúde e no desempenho do trabalhador frente às demandas laborais. Estudo realizado com profissionais de saúde na China por Deng et al. (2021), revelou que o apoio social do supervisor pode reduzir o obstáculo do estresse em trabalhadores. Para os autores essa diminuição do estresse se deveria também ao fato dos trabalhadores perceberem que seus trabalhos são considerados essenciais aos seus supervisores, impactando nas decisões da instituição sobre seu desempenho. Logo, salienta-se que o apoio social é um recurso organizacional importante proporcionado aos trabalhadores, de modo a auxiliá-los a atender as demandas de trabalho.

Outro aspecto observado nas produções de acordo com Kogien e Cedaro (2014) é que as altas demandas quando simultâneas ao controle do trabalhador sobre suas tarefas, associado ao alto apoio social, estes se configuram como um instigador de aprendizado e do crescimento profissional e pessoal. Em contrapartida, quando não ocorre o nível alto de controle, alta demanda e principalmente o apoio social alto no trabalho, isso pode acarretar no prejuízo à saúde do trabalhador.

Por outro lado, trabalhadores que apresentam alta demanda e baixo controle, possuem no seu processo de trabalho características que proporcionam convivência com situações críticas e estimuladoras de estresse, que se torna muitas vezes repetitivas, sem que o profissional tenha autonomia para modificá-lo. Desta forma, torna-se difícil estes trabalhadores serem capazes de proteger sua integridade mental ou física no processo de adoecimento proveniente do desgaste laboral (Velasco, 2014).

Segundo Canesqui e Barsaglini (2012), apoio social sendo um tipo de auxílio que repousa nos intercâmbios e nos padrões de reciprocidade interpessoal nas organizações, depreciação de sua relevância no serviço e na saúde do trabalhador como capital social, gera os efeitos negativos como, a deterioração das condições de saúde desses trabalhadores frente aos eventos deletérios.

Estudo realizado com profissionais da atenção primária, salienta que estes manifestam-se o apoio social adequado em seu ambiente de trabalho, sendo este um aspecto fundamental no autocontrole das situações estressantes percebidas pelos trabalhadores (Santos, et al., 2021).

No entanto, os profissionais de saúde que apresentam em seu trabalho a alta demanda de trabalho, alto controle e o apoio social baixo, representam um trabalho ativo com baixo desgaste. Assim, salienta-se a importância de dar atenção ao baixo apoio social demandado pela instituição a esses trabalhadores (Ribeiro et al., 2018).

O trabalho com alta exigência agrega grandes riscos à saúde do trabalhador, assim como trabalho realizado com altas demandas, mesmo sendo em condições de controle, ainda é capaz de ser prejudicial à saúde psíquica dos profissionais. Quando envolve alta demanda psicológica e baixo controle, o âmbito psicossocial do labor, pode estimular o desgaste prejudicial, com repercussões negativas para saúde mental do trabalhador. Todavia, tais consequências oriundas das demandas do trabalho e do 
controle, representam aspectos que impactam no fenômeno de comparecer ou não no trabalho, mesmo que estes profissionais estejam com alguns problemas de saúde (Shimabuku et al., 2017).

Por esse motivo, é fundamental o desenvolvimento de uma visão atenciosa e sensível aos trabalhadores de saúde, pois o âmbito que estes exercem o cuidado a população e que tem como objetivo a promoção de saúde, muitas vezes, acaba sendo um local gerador de morbidades e perturbações psicológicas a esses profissionais (Oliveira, et al., 2021).

Diante do exposto, ressalta-se que a interação social é um elemento potente que pode propiciar a pessoa a ter noção acerca do suporte social, que se associa proporcionalmente com melhores indicadores da qualidade de vida do trabalhador. Porém, a forma como o trabalho é organizado, pode justificar o nível de baixo apoio social, devido a questão da segregação das equipes de saúde, suprimindo a interação social (Kogien, 2012).

O apoio social, apresenta-se como um importante elemento emocional que influencia a relação demanda-controle, desta forma, serve como amenizador do impacto dos estressores e incrementando a sensação de bem-estar e qualidade de vida dos trabalhadores (Shimabuku et al., 2017). Ainda ressalta a relevância do fortalecimento dos vínculos interpessoais e desenvolvimento de habilidades que possibilitam os profissionais a enfrentarem situações problemáticas do ambiente de trabalho (Candaten, 2015).

Salienta-se a importância de apoio da gestão na manutenção da unidade de saúde, de modo que os trabalhadores possam desempenhar sua função adequadamente com o uso de recursos necessários disponibilizados (Brandão, et al., 2021).

Portanto, torna-se pertinente a ampliação da concepção do suporte social, em especial pelo impacto positivo que pode ter nas políticas de promoção da saúde, nas estratégias e técnicas de enfrentamento da população, na participação social, bem como nas práticas de integralidade do cuidado. Sua manutenção por parte das instituições e gestores, pode contribuir para proteger os trabalhadores dos agravos à sua saúde (Lacerda, 2010; Fernandes et al., 2018).

\section{Considerações Finais}

Por meio do estudo conclui-se que as produções apontam uma tendência da relação do apoio social com à saúde do trabalhador, atuando como fator de proteção a possíveis agravos à saúde do trabalhador, quando presente no ambiente do trabalho ou como meio de equilíbrio da demanda e fortalecimento do controle do trabalhador sobre suas tarefas.

Por outro lado, as produções também sinalizaram os efeitos de apoio social quando baixo entre os trabalhadores o que pode potencializar o desenvolvimento dos agravos relacionados a labor, diminuição da qualidade de vida dos profissionais, bem como esgotamento, desgaste e estresse ocupacional.

Quanto à temática, verificou-se como tendências nas produções o predomínio de delineamento de pesquisa do tipo quantitativo, no cenário hospitalar, na área da enfermagem, em dissertações publicadas entre os anos 2014 a 2018 pertencentes a região sudeste do país. Deste modo, os resultados obtidos neste estudo proporcionaram identificar que ainda há lacunas científicas oriundas das produções nacionais acerca do apoio social entre trabalhadores de APS, sendo uma área importante de investigação. Diante disso, verifica-se a relevância de as pesquisas abordarem a temática nesse contexto de atenção, contribuindo para a saúde do trabalhador, com vistas ao desenvolvimento de estratégias para promover ações que fortalecem o apoio social.

Sugere-se para estudos futuros a investigação da temática de apoio social associada a outros constructos, por exemplo, coping e resiliência ou contrastando-os com agravos em saúde do trabalhador e ainda, investigar outros grupos de trabalhadores. 


\section{Agradecimentos}

Coordenação e Aperfeiçoamento de Pessoal de Nível Superior (CAPES), pela concessão de bolsa de Pós-Graduação mestrado.

\section{Referências}

Andrade, V. P. A., \& Pérez-Nebra, A. R. (2017). Suporte Social e Estresse no Trabalho: uma Análise com Métodos Mistos. Contabilidade, Gestão e Governança. 20(3), 442-462. http://dx.doi.org/10.21714/1984-3925_2017v20n3a7

Andrade, G. R. B., \& Vaitsman, J. (2002). Apoio social e redes: conectando solidariedade e saúde. Ciência \& Saúde Coletiva, 7(4):925-934. https://doi.org/10.1590/S1413-81232002000400023

Baglioli, L. C. S. (2017). A incidência de transtornos mentais e comportamentais nas atividades laborais inclusive sob a percepção da organização internacional do trabalho - OIT. Dissertação de Mestrado. Universidade Católica de Brasília. Brasília, DF. Brasil.

Brasil. (2018). Ministério da Saúde. Secretaria de Atenção à Saúde. Secretaria de Vigilância em Saúde. Saúde do trabalhador e da trabalhadora. Cadernos de Atenção Básica, (41) 136 p.

Brasil. (2001). Ministério da Saúde; Organização Pan-Americana da Saúde (Brasil). Doenças relacionadas ao trabalho: manual de procedimentos para os serviços de saúde. Brasília, DF, Brasil. 508 p.

Brandão, G. C. G., et al. (2021). O Processo de trabalho dos Agentes Comunitários de Saúde. Research, Society and Development. http://dx.doi.org/10.33448/rsd-v10i1.11442

Belini, I. C. (2018). A prática do presenteísmo e suas implicações no cotidiano dos Agentes Comunitários de Saúde. Dissertação de Mestrado. Universidade Federal de Juiz de Fora. Juiz de Fora, MG, Brasil.

Bertoncello, R., Sousa, E., Marques, D. O., \& Oliveira, E. C. (2017). Relações entre Estresse, Saúde Mental e Suporte Organizacional em um Hospital de Ensino. Atas CIAIQ2017. 523-128.

Bezerra, M. L. S., \& Neves, E. B. (2010). Perfil da produção científica em saúde do trabalhador. Saúde Soc. 19(2), 384-394. https://doi.org/10.1590/S010412902010000200014

Brum, C. N., Zuge, S. S., Rangel, R. F., Freitas, H. M. B., \& Pieszak, G. M. (2016). Revisão narrativa da literatura: aspectos conceituais e metodológicos na construção do conhecimento da enfermagem. In: Lacerda, M.R. \& Costenaro, R.G.S. Metodologias da Pesquisa para a Enfermagem e Saúde. Da teoria à prática. Lacerda, M.R. \& Costenaro, R.G.S. (ORG.). 1. Ed. Porto Alegre, RS: Moriá Editora, cap. 2, 51-76.

Canesqui, A. M., \& Barsaglini, R. A. (2012). Apoio social e saúde: pontos de vista das ciências sociais e humanas. Ciência \& Saúde Coletiva, 17(5):11031114. https://doi.org/10.1590/S1413-81232012000500002

Candaten, A. E. (2015). Análise da associação entre a intenção de abandono da profissão e o estresse laboral de enfermeiros de um hospital da Serra Gaúcha/RS. Dissertação de Mestrado. Universidade Federal do Rio Grande do Sul. Porto Alegre, RS. Brasil.

Carneiro, A. S., Andolhe, R., Lanes, T. C., \& Magnago, T. S. B. S. (2020) Cultura de segurança do paciente em ambiente hospitalar: tendências da produção brasileira. Research, Society and Development. 9(7), e963975132. http://dx.doi.org/10.33448/rsd-v9i7.5132

Coimbra, J. R. (2017). Qualidade de vida associada ao estresse no trabalho de profissionais da enfermagem. Dissertação de Mestrado. Universidade do Sagrado Coração. Bauru, SP. Brasil.

Chor, D., Griep, R. H., Lopes, C. S. \& Faerstein, E. (2001). Medidas de rede e apoio social no Estudo Pró-Saúde: pré-testes e estudo piloto. Cad. Saúde Pública. Rio de Janeiro, 17(4), 887-896. https://doi.org/10.1590/S0102-311X2001000400022

Deng, J., Liu, J., Guo, Y., Gao, Y., Wu, Z. \& Yang, T. (2021). How does social support affect public service motivation of healthcare workers in China: the mediating effect of job stress. BMC Public Health. 21:1076. https://doi.org/10.1186/s12889-021-11028-9

Esteves, G. G. L., Leão, A. A. M., \& Alves, E. O. (2019). Fadiga e Estresse como preditores do Burnout em Profissionais da Saúde. Revista Psicologia: Organizações e Trabalho, 19(3), 695-702. http://dx.doi.org/10.17652/rpot/2019.3.16943

Fernandes, G. A. B., Coelho, A. C. O., Paschoalin, H. C., Sarquis, L. M. M. \& Greco, R. M. (2018). Demandas psicológicas, controle e apoio social no trabalho de agentes comunitários de saúde*. Cogitare Enferm. 23(4), e55918. http://dx.doi.org/10.5380/ce.v23i4.55918

Fernandes, G. A. B. (2016). Demandas psicológicas, controle e apoio social no trabalho de agentes comunitários de saúde. Dissertação de Mestrado. Universidade Federal de Juiz De Fora. Juiz de fora, MG. Brasil.

Filho, I.M. M., \& Almeida, R. J. A. (2016). Estresse ocupacional no trabalho em enfermagem no brasil: uma revisão integrativa. Rev Bras Promoç Saúde, Fortaleza, 29(3), 447-454. https://doi.org/10.5020/18061230.2016.p447

Fonseca, I. S. S., \& Moura, S. B. (2008). Apoio social, saúde e trabalho: uma breve revisão. Psicol. Am. Lat. n.15 México. https://www.researchgate.net/publication/317466107

Gadelha, G. O. (2020). Prevalência de sintomas depressivos e de ansiedade e fatores associados em enfermeiros. Dissertação de Mestrado. Universidade Federal do Acre. Rio Branco, Acre. Brasil. 
Johnson, J. V., \& Hall, E. M. Job strain, work place social support, and cardiovascular disease: A cross-sectional study of a random sample of the Swedish working population. Am J Public Health 1988. 78(10): 1336-42.

Junior, E. G., Cardoso, H. F., Domingues, L. C., Green, R. M. \& Lima, T. R. (2014). Trabalho e estresse: identificação do estresse e dos estressores ocupacionais em trabalhadores de uma unidade administrativa de uma instituição pública de ensino superior (IES). Revista GUAL. 7(1), 01-17. http://dx.doi.org/10.5007/1983-4535.2014v7n1p1

Karasek, R., \& Theorell, T. (1990). Healthy work: stress, productivity and the reconstruction of working life. Basic Books.

Kogien, M. (2012). Impactos psicossociais na qualidade de vida de profissionais de saúde em um pronto-socorro público. Dissertação de Mestrado. Fundação Universidade Federal de Rondônia. Porto Velho, Rondônia, Brasil.

Kogien, M., \& Cedaro, J. J. (2014). Pronto-socorro público: impactos psicossociais no domínio físico da qualidade de vida de profissionais de enfermagem. Rev. Latino-Am. Enfermagem. 22(1). 02-08. https://doi.org/10.1590/0104-1169.3171.2387

Lacerda, A. (2010). "Redes de apoio social no Sistema da Dádiva: um novo olhar sobre a integralidade do cuidado no cotidiano de trabalho do agente comunitário de saúde”. Tese de Doutorado. Fundação Oswaldo Cruz. Escola Nacional de Saúde Pública Sergio Arouca. Rio de Janeiro, RJ, Brasil.

Mattos, A. I. S., Araújo, T. M., \& Almeida, M. M. G. (2017). Interação entre demanda-controle e apoio social na ocorrência de transtornos mentais comuns. Rev Saude Publica, 51(0):48. 01-09. https://doi.org/10.1590/S1518-8787.2017051006446

Munhoz, O. L., Andolhe, R., Schimith, M. D., Oliveira, G., Ribeiro, P. L., Silva, T. C. \& Cremonese, L. (2018). Estresse ocupacional e cultura de segurança: tendências para contribuição e construção do conhecimento em enfermagem. ABCS health Sci, 43(2), 110-16. http://dx.doi.org/10.7322/abcshs.v43i2.991.

Oliveira, D. J. B., et al. (2021). Garantias basilares à saúde mental em cenário pandêmico: reflexões sobre as implicações psicológicas nos profissionais de saúde. Research, Society and Development. http://dx.doi.org/10.33448/rsd-v10i11.19566

Polit, D. F., \& Beck, C. T. (2019). Fundamentos de pesquisa em enfermagem: avaliação de evidências para a prática da enfermagem. Artmed.

Ribeiro, R. P., Marziale, M. H. P., Martins, J. T., Galdino, M. J. Q., \& Ribeiro, P. H. V. (2018). Estresse ocupacional entre trabalhadores de saúde de um hospital universitário. Rev Gaúcha Enferm. 39: e65127. 01-06. https://doi.org/10.1590/1983-1447.2018.65127

Rocha, R. P. S. (2018). Características do Trabalho e Estresse Ocupacional entre Enfermeiros no Modelo Clínico Hospitalar. Dissertação de Mestrado. Universidade Federal de Mato Grosso. Cuiabá, MT. Brasil.

Santos, E. C., et al. (2021). Estresse no trabalho em enfermeiros brasileiros atuantes na atenção primária à saúde. Research, Society and Development. http://dx.doi.org/10.33448/rsd-v10i7.13452

Sidone, O. J. G., Haddad, E. A., \& Mena-Chalco, J. P. (2016). A ciência nas regiões brasileiras: evolução da produção e das redes de colaboração científica. TransInformação. Campinas, 28 (1):15-31. http://dx.doi.org/10.1590/2318-08892016002800002

Silva, G. C. R. F. (2010). Método científico na psicologia: abordagem qualitativa e quantitativa. Portal dos Psicólogos.

Silva, A. C. A., \& Silva, A. L. C. (2019). A Educação Continuada e Permanente em Enfermagem no Brasil: uma revisão integrativa. Revista Educação em Saúde. 7 (1): 67 - 73. https://doi.org/10.29237/2358-9868.2019v7i1.p67-73

Silva, A. M. (2014). Estresse ocupacional e qualidade de vida dos profissionais de enfermagem de um Hospital Universitário da cidade de Campo Grande/MS. Dissertação de Mestrado. Universidade Católica Dom Bosco, Brasil.

Shimabuku, R. H., Mendonça, H., \& Fidelis, A. (2017). Presenteísmo: contribuições do Modelo Demanda-Controle para a compreensão do fenômeno. Cadernos de Psicologia Social do Trabalho, 20(1), 65-78. https://doi.org/10.11606/issn.1981-0490.v20i1p65-78

Velasco, A. R. (2014). O estresse laboral dos trabalhadores de enfermagem das equipes de saúde da família. Dissertação de Mestrado. Universidade Federal do Estado do Rio de Janeiro. Brasil. 